\title{
Tracked Regularized Ultrasound Elastography for Targeting Breast Radiotherapy
}

\author{
Hassan Rivaz, Pezhman Foroughi, Ioana Fleming, Richard Zellars, \\ Emad Boctor, and Gregory Hager
}

Johns Hopkins University, Baltimore, MD, USA

\begin{abstract}
Tracked ultrasound elastography can be used for guidance in partial breast radiotherapy by visualizing the hard scar tissue around the lumpectomy cavity. For clinical success, the elastography method needs to be robust to the sources of decorrelation between ultrasound images, specifically fluid motions inside the cavity, change of the appearance of speckles caused by compression or physiologic motions, and out-of-plane motion of the probe. In this paper, we present a novel elastography technique that is based on analytic minimization of a regularized cost function. The cost function incorporates similarity of RF data intensity and displacement continuity, making the method robust to small decorrelations present throughout the image. We also exploit techniques from robust statistics to make the method resistant to large decorrelations caused by sources such as fluid motion. The analytic displacement estimation works in real-time. Moreover, the tracked data, used for targeting the radiotherapy, is exploited for discarding frames with excessive outof-plane motion. Simulation, phantom and patient results are presented.
\end{abstract}

\section{Introduction}

Breast irradiation after lumpectomy significantly reduces the risk of cancer recurrence. There is growing evidence suggesting that irradiation of only the involved area of the breast, partial breast irradiation (PBI), is as effective as whole breast irradiation [1]. Benefits of PBI include significantly shortened treatment time and fewer side effects as less tissue is treated. However, these benefits cannot be realized without localization of the lumpectomy cavity. Tracked ultrasound elastography can be used for localizing the lumpectomy cavity in the treatment room, minimizing tissue motion from planning to treatment.

This paper is focused on freehand palpation elastography, which involves estimating the displacement field of the tissue undergoing slow compression. Most elastography techniques estimate the displacement field using local cross correlation analysis of echoes 234. These methods are very sensitive and accurate for calculating small displacements. However, elastography is subject to speckle decorrelation caused by various sources such as motion of subresolution scatterers, out-of-plane motion, high compression and complex fluid motions.

The prior of tissue deformation continuity can be used to make elastography more robust to signal decorrelation. Previous work on regularized elastography 
is computationally expensive [56]. Dynamic programming (DP) can be used to speed the optimization procedure [7, but it only gives integer displacements. Subpixel displacement estimation is possible [7, but it is computationally expensive if a fine subpixel level is desired. In addition, a fixed regularization weight is applied throughout the image. However, while two ultrasound images may correlate well in most parts, they can have small correlation in specific parts. Four examples of low correlation are: (1) correlation decreases with depth mainly due to a decrease in the ultrasonic signal to noise ratio, (2) correlation is low close to arteries due to complex motion and inside vessels due to blood motion, (3) correlation is extremely low in lesions that contain liquid due to the incoherent fluid motion 83 , and (4) out-of-plane motion of movable structures within the image 8 causes low local correlation. To prevent such regions from introducing errors in the displacement estimation one should use large weights for the regularization term, resulting in over-smoothing.

Freehand palpation elastography provides ease-of-use and requires minimum additional cost. However, out-of-plane motion cannot be avoided in freehand palpation, which reduces the quality of any elastography method. Assisted freehand elastography [9] significantly reduces the out-of-plane motion but it requires addition of a device to the probe. Quality metrics such as persistence in strain images have also been developed to address this problem [10]. To measure the persistence, elastography is performed on two pairs of images and the resulting strain images are correlated. This method requires strain images for calculating the quality metric. Therefore, trying all the combinations in a series of frames to find the best pair for elastography will be computationally expensive.

In this paper, we present a novel elastography method based on analytic minimization (AM) of a cost function that incorporates similarity of echo amplitudes and displacement continuity. We introduce a novel regularization term and demonstrate that it minimizes displacement underestimation caused by smoothness constraint. We also introduce the use of robust statistics implemented via iterated reweighted least squares (IRLS) to treat uncorrelated ultrasound data as outliers. And finally, we use the tracking information to select the best pairs of frames for elastography. Simulation, phantom and patient experiments are presented for validation.

\section{Regularized Displacement Estimation}

Dynamic Programming (DP). DP is a discrete efficient optimization technique for causal systems. In DP elastography [7], a cost function is defined as

$$
C\left(i, d_{i}\right)=\min _{d_{i-1}}\left\{C\left(i-1, d_{i-1}\right)+\alpha_{a} R\left(d_{i}, d_{i-1}\right)\right\}+\left|I_{1}(i)-I_{2}\left(i+d_{i}\right)\right|, i=2 \cdots m
$$

where $d_{i}$ is the displacement of sample $i, R\left(d_{i}, d_{i-1}\right)=\left(d_{i}-d_{i-1}\right)^{2}$ is an axial regularization term (axial, lateral and out-of-plane directions are respectively $z, x$ and $y$ in Figure 2 (a)), $\alpha_{a}$ is a weight for the regularization, $I_{1}$ and $I_{2}$ are corresponding RF-lines of before and after deformation and $m$ is the length 
of RF-lines. The cost function is minimized at $i=m$ and the $d_{i}$ values that have minimized the cost function are traced back to $i=1$, giving the $d_{i}$ for all samples. We have implemented a 2D DP algorithm similar to [7 to generate integer displacements as a starting point for the next step of our algorithm.

Analytic Minimization (AM). We now propose a method that analytically minimizes a regularized cost function and gives the refined displacement field. Only axial displacements will be refined for strain calculation.

Having the integer displacements $d_{i}$ from DP, it is desired to find $\Delta d_{i}$ values such that $d_{i}+\Delta d_{i}$ gives the value of the displacement at the sample $i$ for $i=$ $1 \cdots m$. Such $\Delta d_{i}$ values will minimize the following regularized cost function

$$
\begin{aligned}
& C\left(\Delta d_{1}, \cdots, \Delta d_{m}\right)=\sum_{i=1}^{m}\left[I_{1}(i)-I_{2}\left(i+d_{i}+\Delta d_{i}\right)\right]^{2}+ \\
& \alpha_{a}\left(d_{i}+\Delta d_{i}-d_{i-1}-\Delta d_{i-1}\right)^{2}+\alpha_{l}\left(d_{i}+\Delta d_{i}-d_{i}^{p \cdot}-\Delta d_{i}^{p \cdot}\right)^{2}
\end{aligned}
$$

where superscript $p$. refers to the previous RF-line (adjacent RF-line in the lateral direction) and $\alpha_{l}$ is a weight for lateral regularization. Substituting $I_{2}(i+$ $\left.d_{i}+\Delta d_{i}\right)$ with its first order Taylor expansion approximation around $d_{i}$, we have

$$
\begin{gathered}
\left.C\left(\Delta d_{1}, \cdots, \Delta d_{m}\right)=\sum_{i=1}^{m}\left[I_{1}(i)-I_{2}\left(i+d_{i}\right)-I_{2}^{\prime}\left(i+d_{i}\right) \Delta d_{i}\right)\right]^{2}+ \\
\alpha_{a}\left(d_{i}+\Delta d_{i}-d_{i-1}-\Delta d_{i-1}\right)^{2}+\alpha_{l}\left(d_{i}+\Delta d_{i}-d_{i}^{p .}-\Delta d_{i}^{p \cdot}\right)^{2}
\end{gathered}
$$

where $I_{2}^{\prime}$ is the derivative of the $I_{2}$. The optimal $\Delta d_{i}$ values occur when the partial derivative of $C$ w.r.t. $\Delta d_{i}$ is zero. Setting $\frac{\partial C}{\partial \Delta d_{i}}=0$ we have

$$
\left(\mathbf{I}_{2}^{\prime 2}+\alpha_{a} \mathbf{D}+\alpha_{l} \hat{\mathbf{I}}\right) \Delta \mathbf{d}=\mathbf{I}_{2}^{\prime} \mathbf{e}-\left(\alpha_{a} \mathbf{D}+\alpha_{l} \hat{\mathbf{I}}\right) \mathbf{d}+\alpha_{l} \mathbf{d}^{t . p .}, \quad \mathbf{D}=\left[\begin{array}{ccccc}
1 & -1 & 0 & \cdots & 0 \\
-1 & 2 & -1 & \cdots & 0 \\
\vdots & & & \ddots & \\
0 & \cdots & 0 & -1 & 1
\end{array}\right]
$$

where $\mathbf{I}_{2}^{\prime}=\operatorname{diag}\left(I_{2}^{\prime}\left(1+d_{1}\right) \cdots I_{2}^{\prime}\left(m+d_{m}\right)\right), \Delta \mathbf{d}=\left[\Delta d_{1} \cdots \Delta d_{m}\right]^{T}, \mathbf{e}=\left[e_{1} \cdots e_{m}\right]^{T}$, $e_{i}=I_{1}(i)-I_{2}\left(i+d_{i}\right), \mathbf{d}=\left[d_{1} \cdots d_{m}\right]^{T}, \mathbf{d}^{t \cdot p \cdot}=\mathbf{d}^{p \cdot}+\Delta \mathbf{d}^{p}$ is the vector of total displacement of the previous line and $\hat{\mathbf{I}}$ is the identity matrix. $\mathbf{I}_{2}^{\prime}, \mathbf{D}$ and $\hat{\mathbf{I}}$ are matrices of size $m \times m$ and $\Delta \mathbf{d}, \mathbf{r}, \mathbf{d}$ and $\mathbf{d}^{t . p}$. are vectors of size $m$.

Biasing the Regularization. The regularization term $\alpha_{a}\left(d_{i}+\Delta d_{i}-d_{i-1}-\right.$ $\left.\Delta d_{i-1}\right)^{2}$ penalizes the difference between $d_{i}+\Delta d_{i}$ and $d_{i-1}+\Delta d_{i-1}$, and therefore can result in underestimation of the displacement field. Such underestimation can be prevented by biasing the regularization by $\epsilon$ to $\alpha_{a}\left(d_{i}+\Delta d_{i}-d_{i-1}-\right.$ $\left.\Delta d_{i-1}-\epsilon\right)^{2}$, where $\epsilon=\left(d_{m}-d_{1}\right) /(m-1)$ is the average displacement difference between samples $i$ and $i-1$. An accurate enough estimate of $d_{m}-d_{1}$ is known from the previous line. With the bias term, the R.H.S. of Equation 4 becomes $\mathbf{I}_{2}^{\prime} \mathbf{e}-\left(\alpha_{a} \mathbf{D}+\alpha_{l} \hat{\mathbf{I}}\right) \mathbf{d}+\alpha_{l} \mathbf{d}^{t \cdot p}+\mathbf{b}$ where the bias term is $\mathbf{b}=\alpha_{a}\left[\begin{array}{llll}-\epsilon & 0 & \cdots & \epsilon\end{array}\right]^{T}$ and all other terms are as before. Interestingly, except for the first and the last equation in this system, all other $m-2$ equations are same as Equation 4

Equation 4 can be solved for $\Delta \mathbf{d}$ in $4 \mathrm{~m}$ operations since the coefficient matrix $\mathbf{I}_{2}^{\prime 2}+\alpha_{a} \mathbf{D}+\alpha_{l} \hat{\mathbf{I}}$ is tridiagonal. Utilizing its symmetry, the number of operations 
can be reduced to $2 \mathrm{~m}$. The number of operations required for solving a system with a full coefficient matrix is more than $m^{3} / 3$, significantly more than $2 m$.

Making Tracking Resistant to Outliers. Even with pure axial compression, some regions of the image may move out of the imaging plane and increase the decorrelation. In such parts the confidence of the data term is less and therefore the weight of the regularization term should be increased. The parts of the image with low correlation can be regarded as outliers and therefore a robust estimation technique can limit their effect. Before deriving a robust estimator for $\Delta \mathbf{d}$, we rewrite Equation 3 as $C(\Delta \mathbf{d})=\sum_{i=1}^{m} \rho\left(r_{i}\right)+R(\Delta \mathbf{d})$ where $r_{i}=I_{1}(i)-I_{2}\left(i+d_{i}\right)-I_{2}^{\prime}\left(i+d_{i}\right) \Delta d_{i}, \rho\left(r_{i}\right)=r_{i}^{2}$ and $R$ is the regularization term. The M-estimate of $\Delta \mathbf{d}$ is $\Delta \hat{\mathbf{d}}=\arg \min _{\Delta \mathbf{d}}\left\{\sum_{i=1}^{m} \rho\left(r_{i}\right)+R(\Delta \mathbf{d})\right\}$ where $\rho(u)$ is a robust loss function [11]. The minimization is solved by setting $\frac{\partial C}{\partial \Delta d_{i}}=0$ :

$$
\rho^{\prime}\left(r_{i}\right) \frac{\partial r}{\partial \Delta d_{i}}+\frac{\partial R(\Delta \mathbf{d})}{\partial \Delta d_{i}}=0
$$

A common next step [11] is to introduce a weight function $w$, where $w\left(r_{i}\right) \cdot r_{i}=$ $\rho^{\prime}\left(r_{i}\right)$. This leads to a process known as "iteratively reweighted least squares" (IRLS), which alternates steps of calculating weights $w\left(r_{i}\right)$ for $r_{i}=1 \cdots m$ using the current estimate of $\Delta \mathbf{d}$ and solving Equation 5 to estimate a new $\Delta \mathbf{d}$ with the weights fixed. Among many proposed shapes for $w(\cdot)$, we use [1]

$$
w\left(r_{i}\right)=\left\{\begin{array}{cc}
1 & \left|r_{i}\right|<T \\
\frac{T}{\left|r_{i}\right|} & \left|r_{i}\right|>T
\end{array}\right.
$$

where $T$ is a threshold that can be tuned. A small $T$ will treat many samples as outliers. With the addition of the weight function, Equation 5 becomes

$$
\left(\mathbf{w} \mathbf{I}_{2}^{2}+\alpha \mathbf{D}+\alpha_{2} \hat{\mathbf{I}}\right) \Delta \mathbf{d}=\mathbf{w} \mathbf{I}_{2}^{\prime} \mathbf{e}-\left(\alpha_{1} \mathbf{D}+\alpha_{2} \hat{\mathbf{I}}\right) \mathbf{d}+\alpha_{2} \mathbf{d}^{t \cdot p \cdot}+\mathbf{b}
$$

where $\mathbf{w}=\operatorname{diag}\left(w\left(r_{1}\right) \cdots w\left(r_{m}\right)\right)$. All of the results presented in this work are obtained with one iteration of the above equation unless otherwise specified. Current implementation of the AM algorithm with the IRLS takes $0.015 \mathrm{~s}$ to generate a dense displacement field of size $1300 \times 60$ on a $3.4 \mathrm{GHz}$ P4 CPU(not including the DP run time). The computation time increases linearly with the size of images.

Frame Selection. The ultrasound probe is tracked in navigation/guidance systems to provide spatial information, to generate freehand 3D ultrasound, or to facilitate multi-modality registration. Through a calibration process, the $6 \mathrm{DOF}$ motion of the probe in the sensor coordinate system is transformed into image coordinate system 12. The mean of the absolute motion value of all pixels in $3 \mathrm{D},\left\langle\left|v_{x}\right|\right\rangle,\left\langle\left|v_{y}\right|\right\rangle$ and $\left\langle\left|v_{z}\right|\right\rangle$, can be analytically related to the 6DOF sensor readings using straightforward and efficient geometric computations. For frame $i$ and $j$ to be selected from a sequence of frames for elastography,

$$
Q_{i, i}=k_{x}\left\langle\left|v_{x}\right|\right\rangle^{2}+k_{y}\left\langle\left|v_{y}\right|\right\rangle^{2}+k_{z} \frac{\left\|\left\langle\left|v_{z}\right|\right\rangle-v_{z, o p t}\right\|^{3}}{\left\langle\left|v_{z}\right|\right\rangle+c}
$$


should be minimized where $k_{x}, k_{y}$, and $k_{z}$ are weights for lateral, out-of-plane and axial displacements and $v_{z, o p t}$ is the optimum axial motion. Please refer to 12 for a rationale of the shape this function. Note that the selected pairs are not necessarily consecutive frames. The parameters, $k_{x}, k_{y}, k_{z}, v_{z, o p t}$ and $c$ are manually tuned to $1,2,1,0.7$ and 1 for the AM elastography method.

\section{Simulation, Phantom and Patient Results}

Simulation Results. RF ultrasound data of two phantoms are simulated using Field II [13. The first phantom is $50 \times 10 \times 55 \mathrm{~mm}$ and the second one is $36 \times$ $10 \times 25 \mathrm{~mm}$. They are both made of homogeneous and isotropic material: the first one is uniform and the second one contains a circular hole filled with water, simulating a blood vessel in tissue (Figure 2 (a)). A uniform compression in the $\mathrm{z}$ direction is applied and the $3 \mathrm{D}$ displacement field of the phantom is calculated using ABAQUS finite element package (Providence, RI). The Poisson's ratio is set to $\nu=0.49$ in both phantoms to mimic real tissue, which causes the phantom to deform in $\mathrm{x} \& \mathrm{y}$ directions as a result of the compression in the $\mathrm{z}$ direction.

Respectively $5 \times 10^{5}$ and $1.4 \times 10^{5}$ scatterers with uniform scattering strengths are uniformly distributed in the first and second phantom, ensuring more than 10 scatterers exist in a resolution cell. The scatterers are distributed in the $8 \mathrm{~mm}$ diameter vein also (Figure 2 (a)). To construct deformed ultrasound images, the displacement of all of the scatterers is calculated by interpolating the displacement of the neighboring nodes in the finite element analysis. The parameters of the probe are set to mimic Siemens $5-10 \mathrm{MHz}$ probes. The probe frequency is $7.27 \mathrm{MHz}$, the sampling rate is $40 \mathrm{MHz}$ and the fractional bandwidth is $60 \%$.

The first phantom undergoes uniform compressions in the $\mathrm{z}$ direction to achieve strain levels of $2 \%$ to $14 \%$ in $2 \%$ intervals. Ground truth integer displacement values are used as the initial estimate for AM to decouple the performance of DP from AM. Accurate subpixel displacement field is calculated with $\mathrm{AM}$ and the mean strain values are compared with the ground truth (Figure 1 (a)-(c)). The results are only shown for $2 \%, 4 \%, 8 \%$ and $14 \%$ compression for better visualization. The results with two threshold values for IRLS and without IRLS demonstrate that outlier rejection does not affect the mean strain value, while increasing the regularization weight $\alpha_{a}$ increases underestimation of the displacement. The rate of increase of the underestimation with increasing $\alpha_{a}$ is significantly more with the unbiased regularization (dashed line) as expected.

Significantly higher signal to noise ratio (SNR) [2] values can be achieved with outlier rejection (Figure 1 (d)-(f)) without over-smoothing the image with high $\alpha_{a}$ values. To show the performance of the overall method, the initial integer displacement field is calculated with DP and accurate displacement field is calculated with (Figure 1 (g)-(i)). The SNR values are less than previous case especially at high strain values, where DP results deviates from ground truth.

The second simulation experiment is designed to show the effect of smoothness weight and IRLS threshold on contrast to noise ratio (CNR) 2] when the correlation is lower in parts of the image due to fluid motion. The phantom 


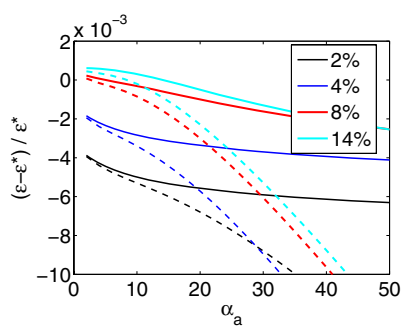

(a) $T=0.005$

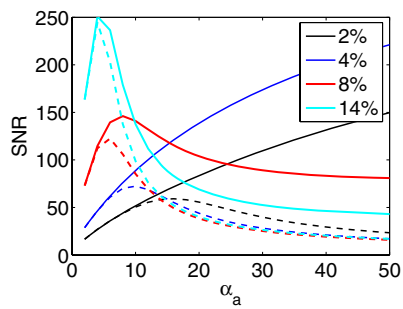

(d) $T=0.005$

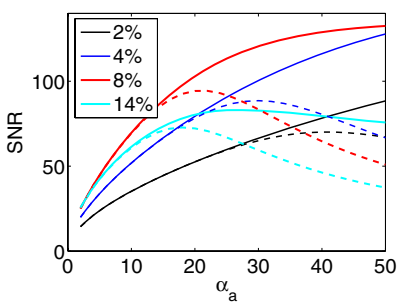

(g) $T=0.005$

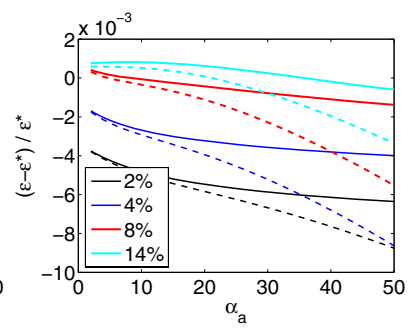

(b) $T=0.01$

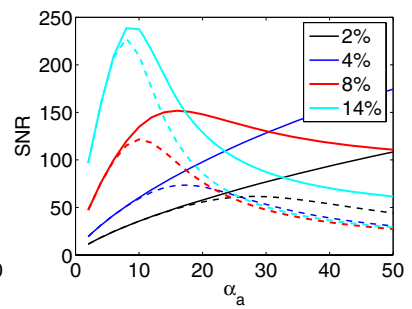

(e) $T=0.01$

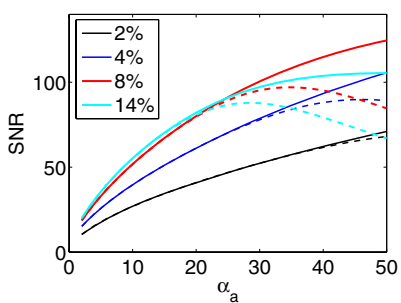

(h) $T=0.01$

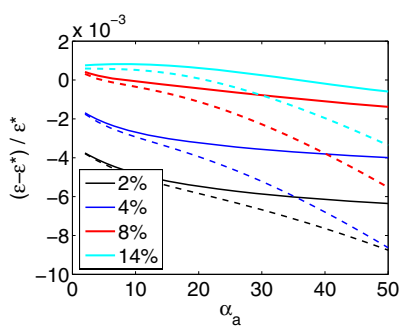

(c) no IRLS

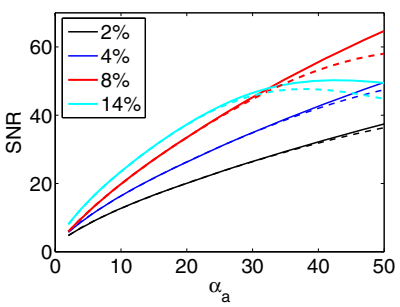

(f) no IRLS



(i) no IRLS

Fig. 1. Mean and SNR of the elastograms of the Field II simulated uniform phantom at four different compression levels (shown in percentage) for three IRLS $T$ values. The solid and dashed lines correspond to biased and unbiased regularizations respectively. (a)-(c) shows the relative underestimation of the strain. $\epsilon$ is the mean strain calculated with the elastography method and $\epsilon^{*}$ is the ground truth. (d)-(f) shows the SNR of the AM. (g)-(i) shows the SNR of the AM with initial displacements found by DP.

contains a vein oriented perpendicular to the image plane (Figure 2). The initial integer displacement is generated with DP. The background window for CNR calculation is located close to the target window to show how fast the strain is allowed to vary, a property related to the spatial resolution. The maximum CNR with IRLS is 5.3 generated at $T=0.005$ and $\alpha_{a}=38$, and without IRLS is 4.8 at $\alpha_{a}=338$. Such high $\alpha_{a}$ value makes the share of the data term in the cost function very small and causes over-smoothing.

Phantom Results. We perform freehand palpation experiment on a breast phantom to examine the performance of the frame selection technique. 50 frames of RF ultrasound data are acquired using a Siemens Antares system (Issaquah, WA). Our custom data acquisition program is connected to the Axius Direct Research Interface to send the command for capturing RF data. At the same 


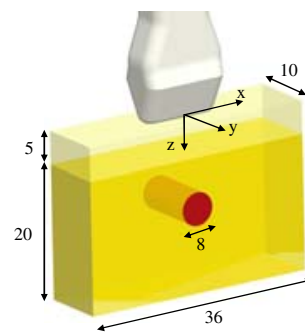

(a) simulation phantom

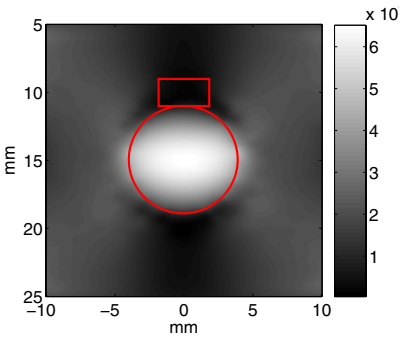

(b) finite element strain

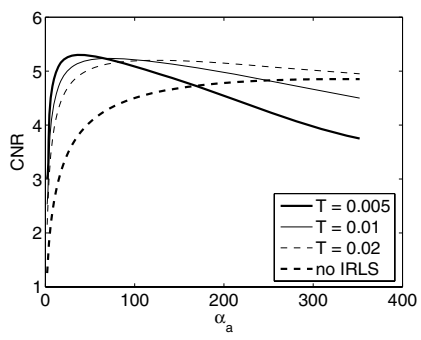

(c) $\mathrm{CNR}$

Fig. 2. The target (circular) and background (rectangular) windows for CNR calculation of (c) are shown in (b)


Fig. 3. The SNR and CNR of the phantom experiment with and without frame selection

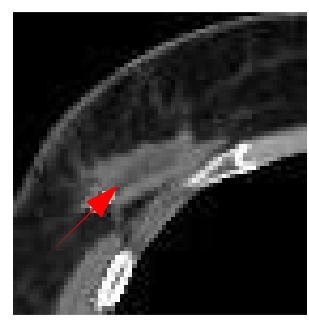

(a) CT image

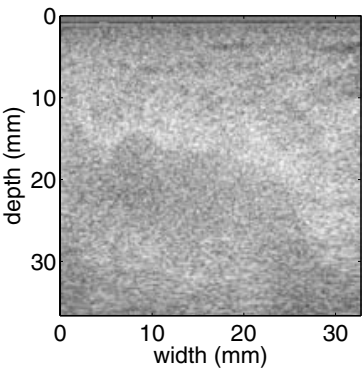

(b) B-mode image

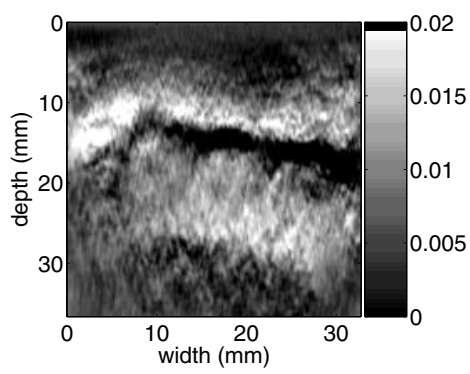

(c) strain image

Fig. 4. Patient experiment results. The arrow points to the lumpectomy cavity.

time, the program collects tracking information from a Polaris tracker (Waterloo, Canada). Currently, the RF frames are stored on the ultrasound system and are processed offline. Figure 3 shows the SNR and CNR results. In automatic frame selection, $Q_{i, j}$ (equation 8) for any two frames $i, j$ in a buffer of size 15 frames is calculated. For the two frames which give the minimum $Q$, the strain image is obtained. The next image is then fed to the buffer, its first image is removed and the frame selection is performed again. The automatic frame selection gives 8 frame pairs for strain calculation (as seen in the figure by 8 SNR and CNR 
values). Without frame selection, 49 strain images are calculated. The average CNR and SNR values are improved from 4.91 to 7.19 and from 4.98 to 5.88 with frame selection.

Patient Results. We have acquired freehand palpation ultrasound RF data using the Siemens Antares system from patients approximately four weeks after lumpectomy. The ultrasound probe is tracked with the Polaris tracking system. Optimal frame selection is performed to select images for elastography using the AM method. The strain image (Figure 4) shows that the AM method can detect the thin hard scar tissue even though it is close to the cavity fluids which undergo incoherent motions and cause signal decorrelation. Since the AM method finds the displacement of all the samples on an A-line at the same time, the correlated data at the top and bottom of the cavity guide the method to find the correct displacement inside the cavity where the data is decorrelated.

\section{Discussion and Conclusion}

We introduced a novel method for calculating a dense displacement map by analytic minimization of a cost function. We used the IRLS method from robust statistics to make the tracking resistant to outliers. Moreover, we exploited the tracking data to optimize frame selection. Through simulation studies using Field II and finite element analysis, we showed that the proposed AM method generates high quality displacement estimates. The elastography method works in real-time. A comparison of the IRLS method with quality guided displacement tracking [14] which also aims for robustness is a subject of future work.

We chose the novel application of the lumpectomy cavity localization as the hard scar tissue is relatively thin and demands a high resolution elastography method. Also, incoherent fluid motions in the cavity causes large decorrelations, requiring a robust method. We have an active Institutional Review Board(IRB) protocol and have promising results from 9 patients which will be published in future work.

Acknowledgments. We would like to thank Shelby Brunke for ultrasound support. Hassan Rivaz is supported by DoD Breast Cancer Research Predoctoral Award and by Link Foundation Fellowship. This work is funded by Breast Cancer Research Foundation, by Siemens Medical Solutions and by CISST ERC NSF EEC-9731748.

\section{References}

1. Offersen, B., Overgaard, M., Kroman, N., Overgaard, J.: Accelerated partial breast irradiation as part of breast conserving therapy of early breast carcinoma: a systematic review. Radiotherapy and Oncology 90(1), 1-13 (2009)

2. Ophir, J., Alam, S., Garra, B., Kallel, F., Konofagou, E., Krouskop, T., Varghese, T.: Elastography. Annu. Rev. Biomed. Eng. 213, 203-233 (1999) 
3. Hall, T., Zhu, Y., Spalding, C.: In vivo real-time freehand palpation imaging. Ultrasound Med. Biol. 29, 427-435 (2003)

4. Zahiri, R., Salcudean, S.: Motion estimation in ultrasound images using time domain cross correlation with prior estimates. Biomed. Eng. 53(10), 1990-2000 (2006)

5. Pellot-Barakat, C., Frouin, F., Insana, M., Herment, A.: Ultrasound elastography based on multiscale estimations of regularized displacement fields. IEEE Trans. Med. Imag. 23(2), 153-163 (2004)

6. Brusseau, E., Kybic, J., Deprez, J., Basset, O.: 2-d locally regularized tissue strain estimation from radio-frequency ultrasound images: Theoretical developements and results on experimental data. IEEE Trans. Med. Imag. 27(2), 145-160 (2008)

7. Rivaz, H., Boctor, E., Foroughi, P., Fichtinger, G., Hager, G.: Ultrasound elastography: a dynamic programing approach. IEEE Trans. Med. Imag 27, 1373-1377 (2008)

8. Lyshchik, A., Higashi, T., Asato, R., et al.: Thyroid gland tumor diagnosis at US elastography. Radiology 237, 202-211 (2005)

9. Kadour, M., Noble, A.: Assisted-freehand ultrasound elasticity imaging. IEEE Trans. Ultrason. Ferroelectr. Freq. Control. 129, 36-43 (2009)

10. Lindop, J., Treece, G., Gee, A., Prager, R.: An intelligent interface for freehand strain imaging. Ultrasound Med. and Biol. 34(7), 1117-1128 (2008)

11. Stewart, C.: Robust parameter estimation in vision. SIAM 41(3), 513-537 (1999)

12. Foroughi, P., Hager, G., Boctor, E.: Robust elasticity imaging using external tracker. In: IEEE Int. Symp. Biomed. Imag. (in press, 2009)

13. Jensen, A.: Field: A program for simulating ultrasound systems. Medical \& Biological Engineering \& Computing 34, 351-353 (1996)

14. Chen, L., Treece, G., Lindop, J., Gee, A., Prager, R.: A quality-guided displacement tracking algorithm ultrasonic elasticity imaging. Med. Imag. A. 13, 286-296 (2009) 